\title{
THE CLOUDS OF M 82
}

P. SCHILKE ${ }^{1}$

Max-Planck-Institut für Radioastronomie, Auf dem Hügel 69, D-5300 Bonn 1, F.R. Germany

N. BROUILLET AND A. BAUDRY

Observatoire de Bordeaux, B.P. 89, F-33270 Floirac, France

\section{INTRODUCTION}

All the existing molecular maps of M82 made up to now have revealed a doublelobed stucture interpreted as a uniform torus (Nakai et al. 1987). We have observed the central part of M 82 in the $\operatorname{HCN}(J=1-0)$ line with the Plateau de Bure IRAM interferometer (spatial resolution $=2.5^{\prime \prime}$ ). The HCN molecule, due to its high dipole moment, traces clumps with a density of about $10^{5} \mathrm{~cm}^{-3}$. For technical reasons we have divided our study in two fields. We present here the results obtained in the south-west field, covering one lobe of the "molecular ring" and the center of the galaxy in the velocity range $0-260 \mathrm{~km} \mathrm{~s}^{-1}$.

\section{RESULTS}

The HCN channel maps reveal the clumpy structure of the molecular gas on a $3^{\prime \prime}$ scale (i.e. $50 \mathrm{pc}$ ). Five peaks of emission are resolved on the line integrated map (see Fig. 1). The clump P4, located near the center of the galaxy, is particularly outstanding; it appears as an intense and compact (spatially unresolved) complex with a very extended velocity range $\left(\approx 100 \mathrm{~km} \mathrm{~s}^{-1}\right)$. Another striking result is the anticorrelation between the spatial distribution of the strong supernova remnants (radio sources of Kronberg et al. 1985) and the dense molecular clouds (Fig. 1). Models of star formation and evolution would predict a coexistence rather than supernova remnants encircling molecular clouds, as observed. Using LVG calculations and comparing our results with the $\mathrm{HCN}(3-2)$ observations of Wild et al. (1992), we derive $\mathrm{N}(\mathrm{HCN}) \approx 210^{15} \mathrm{~cm}^{2}$ and $n\left(\mathrm{H}_{2}\right) \approx 310^{5} \mathrm{~cm}^{-3}$ for $T_{\mathrm{kin}}=50 \mathrm{~K}$ and typical linewidths of $\approx 100 \mathrm{~km} \mathrm{~s}^{-1}$.

\section{THE INTERSTELLAR MEDIUM OF M 82}

We have analysed the spatial structure of our maps using a procedure which fits gaussian clumps in the $\alpha-\delta-v$ cube as in Stutzki and Güsten (1990). Clump masses range from $210^{5} \mathrm{M}_{\odot}$ to $410^{6} \mathrm{M}_{\odot}$. We find a very high fraction of dense

\footnotetext{
${ }^{1}$ now at California Institute of Technology, Division of Physics, Mathematics and Astronomy, 320-47, Pasadena, CA 91125, USA
} 


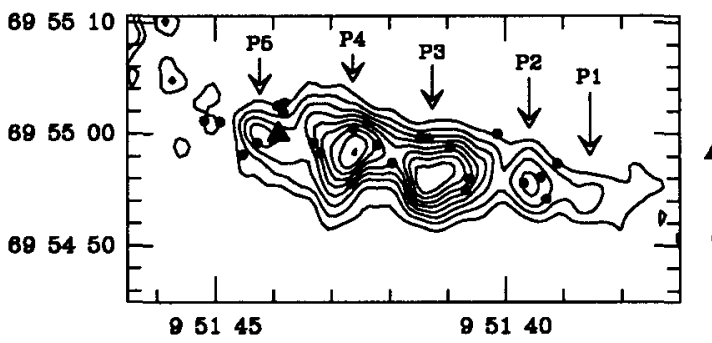

First level: $8 \mathrm{mJy} \cdot \mathrm{km} / \mathrm{s}$

Contour interval: $5 \mathrm{mJy} . \mathrm{km} / \mathrm{s}$

$\Delta \quad 2.2 \mu \mathrm{m}$ peak of Rieke et al. (1980, ApJ 238, 24)

- Radio point sources of Kronberg et al. (1985)

FIGURE I Integrated HCN(1-0) map of the SW-field of M 82. This map covers only the velocity range $0-260 \mathrm{~km} \mathrm{~s}^{-1}$. The five primary peaks are indicated.

gas mass to total gas mass (44\%). We explain this lack of low density molecular gas by the fact that the interclump medium is dissociated and ionized by the very high UV field of M 82 and/or shocks induced by stellar winds and supernova remnants. This view is further supported by the strong spatial correlation of the molecular and ionized gas components (since the continuum emission at $3.4 \mathrm{~mm}$ appears to be due to free-free emission, cf. Carlstrom and Kronberg 1991). This correlation is neither observed in our Galaxy nor in IC 342 (Downes et al. 1992). Our data strongly suggest that the $\mathrm{HCN}$ emission comes from many dense cores (with sizes of $0.001-0.1 \mathrm{pc}$ and masses of $0.006-0.6 \mathrm{M}_{\odot}$ ), surrounded by envelopes of photodissociated dense neutral gas which are embedded in moderately dense ionized gas.

HCN observations of the north-east field of M 82 are in progress. We are also observing the isomer $\mathrm{HNC}$, since the $\mathrm{HCN} / \mathrm{HNC}$ ratio should enable us to estimate the amount of shock- or UV-heated gas. Preliminary $\mathrm{HCN} / \mathrm{HNC}$ results obtained with the IRAM $30 \mathrm{~m}$ radiotelescope show a line ratio $\geq 2$, similar to the one observed towards galactic star forming regions (Schilke et al. 1992).

\section{REFERENCES}

Carlstrom J.E., Kronberg P.P. 1991, Astrophys. J., 366, 422

Downes D., Radford, S.J.E., Guilloteau S., Guélin M., Greve A., Morris D. 1992, $A B A, \mathbf{2 6 2}, 424$

Kronberg P.P., Biermann P., Schwab F.R. 1985, Astrophys. J. , 291, 693

Nakai N., Hayashi M, Handa T., Sofue T., Hasegawa T. 1987, PASJ,39, 685

Schilke P., Walmsley C.M., Pineau des Forêts G., Roueff E., Flower D.R., Guilloteau S. 1992, $A \mathscr{E} A, \mathbf{2 5 6}, 595$

Stutzki J., Güsten R. 1990, Astrophys. J., 356, 513

Wild W., Harris A.I., Eckart A., Genzel R., Graf U.U., Jackson J.M., Russell A.P.G., Stutzki J. 1992, $A \& A$, submitted 